\title{
Síndrome Kawasaki temporalmente relacionado a COVID-19 (Síndrome Kawasaki- Like) en pacientes pediátricos
}

\section{Mucocutaneous Lymph Node Syndrome temporally related to COVID-19 (Kawasaki-like Syndrome) in Pediatric Patients}

Karen Eliana Calderón-Baldera ${ }^{1, \mathrm{a}}$, Walter Luis Gutierrez Celestino-Segura, ${ }^{1, \mathrm{~b}}$

\begin{abstract}
RESUMEN
Introducción: El Síndrome Multisistémico Inflamatorio surge como manifestación de COVID-19 en la edad pediátrica, con diversos fenotipos dentro de los cuales se encuentra un Síndrome similar a Kawasaki, que responde a terapéutica habitual para la enfermedad clásica. Reporte de casos: Se reportan dos casos procedentes de la ciudad de Chiclayo, con edades de 7 y 9 años, sexo femenino, historia de contactos probables y confirmados para COVID-19, y una de las pacientes con serología positiva IgG e imágenes tomográficas compatibles con COVID-19 en resolución. Ambas cumplieron con criterios de Síndrome Multisistémico Inflamatorio Pediátrico y Enfermedad de Kawasaki clásica, además con respuesta favorable a terapéutica habitual, sin complicaciones y en seguimiento actual. Conclusiones: los casos reportados responden a terapéutica habitual y las características de presentación contribuirán a la documentación de dicha patología emergente en la comunidad científica.
\end{abstract}

Keywords: Síndrome Mucocutáneo Linfonodular; Infección por coronavirus; Pediatría(Source: DeCS-BIREME).

\begin{abstract}
ABSTRACS
Introduction: The Inflammatory Multisystemic Syndrome arises as a manifestation of COVID-19 in the pediatric age, with diverse phenotypes within which a Kawasaki-like Syndrome is found, which responds to the usual therapy for classic disease. Case report: Two cases from Chiclayo city, 7 and 9 years old, female sex, history of probable and confirmed contacts for COVID19 , and one of the patients with positive IgG serology and compatible tomographic images with COVID-19 in resolution. Both met criteria for Pediatric Inflammatory Multisystemic Syndrome and classic Kawasaki Disease, in addition with a favorable response to usual therapy, without complications and in current follow-up. Conclusions: the reported cases respond to usual therapy and the presentation characteristics will contribute to the documentation of this emerging pathology in the scientific community.
\end{abstract}

Keywords: Mucocutaneous Lymph Node Syndrome; Coronavirus infections; Pediatric (Source: DeCSBIREME).

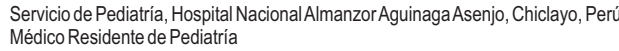

\section{INTRODUCCIÓN}

La enfermedad por coronavirus (SARS-CoV-2) denominada COVID-19, reporta sus primeros casos en diciembre 2019 en la ciudad de Wuhan, se ha extendido por todo el mundo, siendo declarada pandemia por la OMS el 11 de marzo del $2020^{(1)}$. Hasta la actualidad su incidencia en menores de 18 años (edad pediátrica) es baja, en el mundo el número de casos reportados es el $1-5 \%$ del total ${ }^{(1)}$.

En el Perú se han reportado una incidencia de 4,3\% al 29 de Junio del $2020^{(2)}$, cifra que es alta en relación a otros países como Estados Unidos que reporta $1,7 \%{ }^{(3)}$. En la Red Lambayeque de la Seguridad Social (ESSALUD), hasta la Semana Epidemiológica 30, se registraron 4887 casos positivos de COVID-19, de los cuales el 3\% fueron menores de 18 años ${ }^{(4)}$.

Según la evidencia reportada en población pediátrica aunque la mayoría presenta síntomas leves, el 26 de Abril de 2020 en Reino Unido y a principios de Mayo en EEUU pone en alerta a la sociedad científica la aparición creciente de casos de síndrome inflamatorio severo con características similares a enfermedad de Kawasaki (EK) en niños previamente sanos con clínica de COVID-19 en días previos y/o prueba rápida $\operatorname{lgG}^{(5)}$, 
con síntomas respiratorios leves o sin sintomatología respiratoria, que responde a terapia usada para dicha enfermedad (inmunoglobulina y ácido acetilsalicílico). Ante la aparición de dicha patología y al desconocer si se trata de la EK descrita originalmente, el 14 de Mayo de 2020, la CDC (Centers for Disease Control and Prevention) lo denomina como Síndrome de respuesta inflamatoria sistémica pediátrica (PIM-S, o MIS-C), determinando criterios de sospecha que no necesita los criterios rigurosos y típicos utilizados para la enfermedad de Kawasaki Clásica (EKC) $)^{(6,7)}$.

Con los reportes de casos, hasta ahora se sabe que el PIM-S probablemente es un espectro de enfermedad que posee fenotipos como enfermedad similar a Kawasaki, Síndrome de activación de macrófagos y Síndrome de Shock Toxico ${ }^{4,8}$.

En Perú los primeros casos de Síndrome de Kawasaki posteriores de COVID-19 reportados datan del 1 de Junio de $2020^{(9-11)}$

\section{REPORTE DEL CASO}

\section{CASO CLÍNICO 01}

Paciente sexo femenino de 9 años de edad, procedente de Chiclayo-Lambayeque-Perú.

\section{Antecedentes}

Sin antecedentes perinatales patológicos, crecimiento y desarrollo adecuados, esquema de vacunación completo excepto dosis de influenza anual, con antecedentes familiares de dos hermanos con rinitis alérgica ( 22 y 14 años), y patologías previas: alergia a la proteína de leche de vaca y empleo de Salbutamol solo durante el tercer año de vida.

Cuarenta días antes del ingreso, paciente y familiares que comparten vivienda tuvieron síntomas clínicos compatibles con COVID-19, uno de los integrantes fue positivo por prueba rápida (IgG), fueron tratados con Ivermectina y Azitromicina. Ninguno requirió ni tuvo criterios de hospitalización.

\section{Enfermedad actual:}

Paciente inicia enfermedad con dolor abdominal difuso y fiebre $\left(38,2^{\circ}\right)$, recibe sin indicación médica: sulfametoxazol trimetropim por 2 días, en su tercer día de enfermedad persiste febril con picos máximos de $40^{\circ} \mathrm{C}$ cada 6-8 horas, por lo que acude a consulta médica particular, donde le solicitan exámenes auxiliares (realizados en el $4^{\circ}$ día de enfermedad) (tabla $N^{\circ} 1$ ) evidenciándose en el hemograma: leucocitosis, neutrofilia y linfopenia, reacción inflamatoria en heces negativa y serología COVID-19: IgG positiva, siendo medicado con amoxicilina/acido clavulánico por probable infección gastrointestinal. El mismo día antes de recibir la medicación se agrega exantema morbiliforme tipo maculo papular pruriginoso en tórax y abdomen, el cual dos días después se extienden a espalda, axilas y cuello. Por persistencia de dolor abdominal y fiebre realiza nueva consulta médica particular en su quinto día de enfermedad recibiendo azitromicina (dos dosis), al día siguiente disminuye el dolor abdominal pero por persistencia de prurito y fiebre acude a emergencia pediátrica de nuestro hospital, recibiendo manejo de urticaria; exámenes auxiliares dentro de los cuales resalta PCR:13,5 mg/dl (valor normal $<2 \mathrm{mg} / \mathrm{dl}$ ), examen de orina no patológico, urocultivo negativo, coprocultivo positivo a enterobacter cloacae, hemocultivo negativo, transaminasas normales, perfil de coagulación no alterado, tomografía torácica informada: tenues opacidades parcheadas de distribución subpreural en lóbulos inferiores a predominio izquierdo, que puede estar en relación a proceso inflamatorio temprano o en resolución $(D / C$ SARS-COV-2). Al cursar con estabilidad clínica, mejoría de dolor abdominal y de lesiones dérmicas es dada de alta.

En su séptimo día de enfermedad persiste febril y se añade inyección conjuntival bilateral no purulenta, dolor en región cervical anterior, malestar general, artralgias y odinofagia, reingresando al siguiente día en su 8 octavo día de enfermedad al servicio de emergencia pediátrica de nuestro hospital.

A su ingreso en el examen físico de la paciente se evidencian adenopatías cervicales bilaterales $1,5 \mathrm{~cm}$, inyección conjuntival ya descrita, orofaringe congestiva no exudativa, labios agrietados no lesiones dérmicas, neurológicamente alerta, conectada con su entorno sin déficit motor, tono muscular adecuado. En sus resultados de laboratorio resalta leucocitosis con linfopenia, anemia leve, trombocitosis, PCR: $11 \mathrm{mg} / \mathrm{dl}$ (valor normal $<2 \mathrm{mg} / \mathrm{dl}$ ), VSG: $25 \mathrm{~mm} / \mathrm{h}$ (valor normal $<10 \mathrm{~mm} / \mathrm{h}$ ), examen de orina, urocultivo, hemocultivo y coprocultivos no patológicos, perfil hepático normal, perfil de coagulación normal. Por cumplir criterios de EK se realiza ecocardiograma, en el cual no se encontró dilatación de arterias coronarias; además se inicia dosis de Inmunoglobulina, ácido acetilsalicitico (AAS), y hasta resultados de cultivos ceftriaxona.

Paciente presenta ultimo pico febril durante infusión de Inmunoglobulina, permaneciendo afebril $>48$ horas por lo que se disminuye AAS a dosis antiagregante, se suspende ceftriaxona por cultivos negativos y caída de fiebre. Paciente presenta evolución favorable de estado general, caída de valores de reactantes de fase aguda no presenta alteraciones de perfil de coagulación ni hepático durante todo el tiempo de enfermedad. En su día 14 de enfermedad se le realiza control ecocardiográfico no encontrándose dilatación coronaria, ni de aorta ni cayado aórtico, función ventricular conservada siendo dada de alta hospitalaria para seguimiento por consultorio externo. Presenta 
evolución favorable hasta el momento de redacción de CASO CLíNICO 2

este reporte.

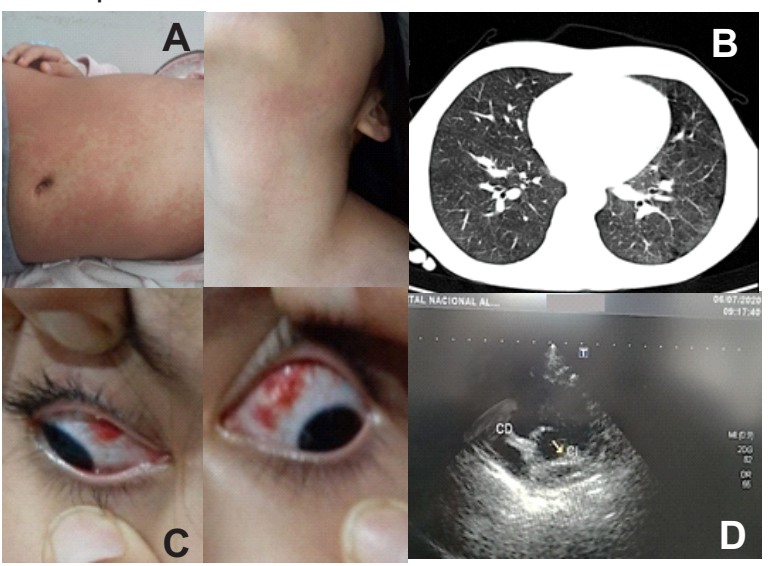

Figura 1

A) Exantema morbiliforme en tórax, abdomen y cuello. B) Tomografía torácica C) Inyección conjuntival bilateral no supurativa D) Ecocardiograma del día 14 de enfermedad: no dilatación de arterias coronarias CD (coronaria derecha), $\mathrm{CI}$ (coronaria izquierda).

Tabla 1. Evolución laboratorial según tiempo de enfermedad.

\begin{tabular}{lccccc}
\hline \multirow{2}{*}{ LABORATORIO } & \multicolumn{5}{c}{ ENFERMEDAD ACTUAL DE CASO 1 } \\
& DIA 4 & DIA 5 & DIA 7 & DIA 10 & DIA 15 \\
\hline Leucocitos & 12010 & 9020 & 20,92 & 4500 & 5790 \\
Linfocitos & $12 \%$ & $13 \%$ & $8 \%$ & $50,7 \%$ & $89 \%$ \\
Neutrófilos & $81 \%$ & $84 \%$ & $91 \%$ & $41,6 \%$ & $10 \%$ \\
Abastonados & $1 \%$ & $0 \%$ & $8 \%$ & $0 \%$ & $0 \%$ \\
Hemoglobina & 11,8 & 11,4 & 10,9 & 9,9 & 10,7 \\
Plaquetas & 254000 & 269000 & 374000 & 510000 & 641000 \\
PCR & - & $13,5 \mathrm{mg} / \mathrm{dl}$ & $11,9 \mathrm{mg} / \mathrm{dl}$ & $6,9 \mathrm{mg} / \mathrm{dl}$ & $1,3 \mathrm{mg} / \mathrm{dl}$ \\
VSG & - & - & $25 \mathrm{~mm} / \mathrm{h}$ & $60 \mathrm{~mm} / \mathrm{h}$ & $57 \mathrm{~mm} / \mathrm{h}$ \\
Dímero D & - & $4,84 \mathrm{ug} / \mathrm{ml}$ & - & - & $2 \mathrm{ug} / \mathrm{ml}$ \\
Troponinas & - & 0,001 & - & - & - \\
CPK-total & - & $39 \mathrm{U} / \mathrm{l}$ & - & - & $21,9 \mathrm{U} / \mathrm{L}$ \\
Albumina & - & - & $3,7 \mathrm{~g} / \mathrm{dL}$ & $3,2 \mathrm{~g} / \mathrm{dL}$ & $3,5 \mathrm{~g} / \mathrm{dL}$ \\
\hline
\end{tabular}

\begin{tabular}{lcccc}
\hline \multirow{2}{*}{ Laboratorio } & ENFERMEDAD ACTUAL DE CASO 2 & \\
& DIA 7 & DIA 9 & DIA 13 & DIA 18 \\
\hline Leucocitos & 11400 & 2900 & 3180 & 5270 \\
Linfocitos & $32 \%$ & $56,9 \%$ & $67 \%$ & $73,5 \%$ \\
Neutrófilos & $57 \%$ & $25,8 \%$ & $22,8 \%$ & $17,1 \%$ \\
Abastonados & $0 \%$ & $0 \%$ & $0 \%$ & $0 \%$ \\
Hemoglobina & 11,4 & 9,5 & 12,3 & 12,6 \\
Plaquetas & 527000 & 476000 & 469000 & 299000 \\
PCR & - & $2 \mathrm{mg} / \mathrm{dl}$ & $0,5 \mathrm{mg} / \mathrm{dl}$ & $0,1 \mathrm{mg} / \mathrm{dl}$ \\
VSG & - & $60 \mathrm{~mm} / \mathrm{h}$ & - & $25 \mathrm{~mm} / \mathrm{h}$ \\
Dímero D & - & $0,800 \mathrm{ug} / \mathrm{ml}$ & - & - \\
LDH & - & 0,001 & - & - \\
CPK-total & - & $56,1 \mathrm{U} / \mathrm{L}$ & - & - \\
Albumina & $3,8 \mathrm{~g} / \mathrm{dL}$ & $3 \mathrm{~g} / \mathrm{dL}$ & $3,7 \mathrm{~g} / \mathrm{dL}$ & - \\
Ferritina & & $251,3 \mathrm{ng} / \mathrm{ml}$ & $530,4 \mathrm{ng} / \mathrm{ml}$ & - \\
\hline
\end{tabular}

Paciente sexo femenino de 7 años, procedente de Chiclayo, Lambayeque, Perú.

\section{Antecedentes}

Nacida por parto por cesárea por Preeclampsia a las 31 semanas de edad gestacional, crecimiento y desarrollo adecuados, esquema de vacunación incompleto, no influenza anual, estreñimiento crónico, antecedentes familiares y personales de atopia: padre asmático, mama con rinitis alérgica, paciente con rinitis alérgica, uso de salbutamol desde el año de vida hasta hace 1 año con frecuencia de 2 veces al año.

Treinta días antes del inicio del cuadro clínico de paciente, miembros de familia con los que parte vivienda presentaron síntomas sugerentes de infección por COVID-19. Uno de ellos con prueba molecular positiva, algunos de ellos recibieron azitromicina e ivermectina, ninguno requirió hospitalización.

\section{Enfermedad actual.}

Paciente ingresa por emergencia con un tiempo de enfermedad de 7 días. Inicia con fiebre $\left(40^{\circ} \mathrm{C}\right)$ que cede parcialmente con paracetamol, adenopatías cervicales bilaterales la mayor de $2 \times 2 \mathrm{~cm}$, dolor abdominal, deposiciones liquidas, hiporexia, malestar general. Por dichos síntomas consultan a medico particular quien indica amoxicilina acido clavulánico, que toma hasta su ingreso a este hospital. Con los días se agregan labios agrietados y eritematosos, lengua en fresa (Figura 2A y $B)$, luego vómitos, náuseas, eritema y descamación perianal (Figura 2C), rash eritematoso escaso no pruriginoso en tórax (Figura 2D). Tres días antes de ingreso se agrega edema y eritema en pies, inyección conjuntival bilateral no purulenta.

Por persistir febril acude por emergencia de nuestro nosocomio. A su ingreso presenta los signos clínicos ya descritos, y al cumplir criterios de EK recibe infusión de inmunoglobulina $2 \mathrm{~g} / \mathrm{kg}$ y AAS $80 \mathrm{mg} / \mathrm{kg} /$ día. Por otro lado, se le realizo prueba rápida COVID-19 resultando no reactiva.

Se hospitaliza en donde se realiza tomografía toráxica informada como normal. Último episodio febril fue horas antes de infusión de inmunoglobulina, por lo que pasadas las 48 horas de finalizada la infusión se disminuye dosis de AAS a $4 \mathrm{mg} / \mathrm{kg} /$ día. Desaparece paulatinamente sintomatología clínica y alteraciones laboratoriales (tabla 2). No presenta alteraciones en ecocardiograma realizados en el día 8 y 12 de enfermedad.

Paciente con evolución muy favorable al alta y hasta el momento de redactar este reporte. 

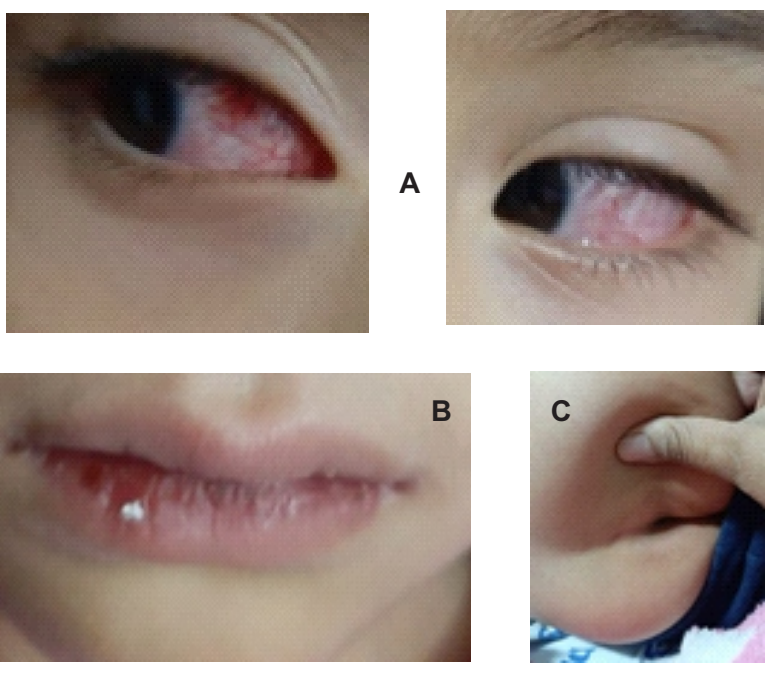

Figura $\mathrm{N}^{\circ} 2$

A) Inyección conjuntival B) Labios agrietados C) Descamación perianal

\section{DISCUSIÓN}

La aparición creciente de casos compatibles con EK en las poblaciones con mayores número de casos de COVID19 puso en alerta a la comunidad científica ante una posible asociación de causalidad entre el virus SARSCOV-2 y la EK, estando a favor la ausencia de etiología definida de esta última, pese 50 años de búsqueda de causalidad viral ${ }^{(8)}$. La hipótesis patogénica más aceptada respalda una respuesta aberrante del sistema inmune a uno o más patógenos no identificados en sujetos genéticamente predispuestos.

Estudios actuales intentan hallar la relación especifica del virus SARS-COV-2 y la EK; postulan la hipótesis de fenómeno post-infeccioso (presencia en días anteriores de síntomas o contacto COVID-19 y el hallazgo de serología IgG positiva) ${ }^{(5)}$. Por otro lado se investiga centra en la proteína $S$ propia de este virus (muy parecida a los superantígenos bacterianos) que posee alta afinidad por los receptores de células T, y genera una respuesta inmune que podría explicar el potencial único del SARS-COV-2 al desarrollo de MIS-C ${ }^{(9)}$. Pese a todo ello, aun su asociación real no está determinada y es producto de investigación hasta la actualidad ${ }^{(10)}$.

En Mayo 2020 las Royal College of Paediatrics and Child Health, Center for disease control y la World Health Organization establecieron criterios para definición de caso de síndrome multisistémico Inflamatorio asociado a COVID-19, los cuales son: fiebre $>38^{\circ} \mathrm{C} \geq 3$ días, disfunción de uno o más órganos, elevación de reactantes inflamatorios (proteína $C$ reactiva (PCR), procalcitonina (PCT), velocidad de sedimentación globular (VSG), serología o PCR positivos para COVID-19 dentro de 4 semanas previo al inicio de síntomas y/o
Contactos probables con pacientes COVID-19, todo esto sumado a 2 o más de los siguientes: Rash o conjuntivitis bilateral no purulenta o signos de inflamación mucocutánea, hipotensión o shock, compromiso cardíaco, evidencia de coagulopatía, compromiso gastrointestinal agudo, elevación de marcadores de inflamación: Procalcitonina, Velocidad de sedimentación globular ${ }^{(11)}$. Ambos casos reportados presentados presentaron fiebre $>3$ días, todos los reactantes de fase aguda elevados en el primer caso al inicio, y solo VSG elevado en el segundo caso, serología positiva IgG positiva en la primera paciente y contactos COVID-19 en ambos casos. Esto último compatible con infección COVID-19 pasada reciente, lo cual es similar a los estudios en un hospital universitario de Francia ${ }^{(5)}$. Sin embargo hay reportes en lo que los pacientes han resultado con infección actual ${ }^{(12)}$, otros en los que ha sido actual y pasada ${ }^{(13)}$, y por ultimo casos no reactivos como nuestra segunda paciente pero con contactos positivos $^{(14)}$.

El diagnostico de EKC se considera en pacientes cuando presentan fiebre durante mínimo 5 días junto a 4 de los siguientes síntomas: 1) Inyección conjuntival, bilateral, no supurativa, 2) Exantema polimorfo generalizado, morbiliforme, confluyente, no vesiculoso, 3) Descamación, eritema y edema de palmas y plantas, 4) Eritemato orofaríngeo con grietas en labios, lengua en fresa, 5) Linfadenopatías cervicales $(>1,5 \mathrm{~cm})$, en ausencia de un diagnostico alternativo. Por otro lado la presentación atípica requiere al menos cinco días de fiebre, menos de 4 de los síntomas mencionados antes pero alteraciones en las arterias coronarias ${ }^{(15-18)}$.

En el caso de nuestras pacientes ambas cumplen criterios de EKC, respondieron a terapéutica a dosis única de Inmunoglobulina, pero están fuera de la edad de presentación habitual, además presentan predominio de dolor abdominal, lo que se ha visto frecuentemente en EK como fenotipo de MIS-C. Sin embargo ninguna presentó disfunción miocárdica que es frecuente en este contexto ${ }^{(15)}$.

Tras la observación de aumento de casos de EK en las zonas más afectadas por la COVID-19 a nivel mundial, se plantea la relación de causalidad que aún está en estudio. Al no ser ajenos a esta realidad, nuestra región Lambayeque una de las más afectadas, se empiezan a presentar casos en la población adscrita a nuestro hospital. Ambas pacientes compatibles con Síndrome Inflamatorio Multisistémico Pediátrico fenotipo EK que respondieron satisfactoriamente a la terapéutica habitual, sin complicaciones durante su hospitalización y al alta, y con seguimiento clínico imagenológico en curso. Se continuará en alerta ante posibles nuevos casos.

Tras la observación de aumento de casos de Enfermedad de Kawasaki en las zonas más afectadas 
por la COVID-19 a nivel mundial, se plantea la relación de causalidad que aún está en estudio. Al no ser ajenos a esta realidad, en nuestra región Lambayeque comienzan a presentarse casos en la población pediátrica adscrita a nuestro hospital. Ambos casos reportados fueron compatibles con Síndrome Inflamatorio Multisistémico Pediátrico fenotipo Enfermedad similar a Kawasaki que respondieron satisfactoriamente a la terapéutica habitual, sin complicaciones durante su hospitalización y al alta, y con seguimiento clínico imagenológico en curso. Cabe mencionar la importancia de documentar los casos que se presenten y así contribuir a la investigación científica.

\section{Agradecimientos}

Al Dr Marco Antonio Aza (Cardio pediatra) por su asesoría temática.

Al Dr Roberto Neciosup Salazar por el apoyo y respaldo como jefe encargado del Servicio de Pediatría del HNAAA.

Al Dr Cristian Diaz Vélez y al Dr Jorge Luis Fernández Mogollón por su asesoría metodológica en la elaboración del presente manuscrito.

A Delia Porras Linaja y Wilder Diaz Rabanal, residentes del servicio quienes brindaron su apoyo en la recolección de datos y búsqueda de información para la redacción del presente.

Conflictos de interés: Los autores niegan conflictos de interés.

Financiamiento: Autofinanciado.

\section{REFERENCIAS BIBLIOGRÁFICAS}

1. Sánchez-Tauma PJ, Atamari-Anahui N, ValeraMoreno C. Enfermedad por Coronavirus 2019, COVID-19: Aspectos a considerar en niños. Rev Cuerpo Méd HNAAA. 2020;13(1):88-94.

2. Centro Nacional de Eidemiologia, Prevención y Control de Enfermedades. Sala Situacional «COVID-19» al 21 de Junio 2020. [Internet]. Lima: Ministerio de Salud; 2020 de unio del [citado 7 de julio de 2020] p. 30. Disponible en: https://www.dge.gob.pe/portal/docs/tools/cor onavirus/coronavirus290620.pdf

3. Covid CDC, COVID C, COVID C, Bialek S, Gierke R, Hughes M, et al. Coronavirus Disease 2019 in Children-United States, February 12-April 2, 2020. Morb Mortal Wkly Rep. 2020;69(14):422.

4. ESSALUD. HOSPITAL NACIONAL ALMANZOR AGUINAGA ASENJO ESSALUD. OFICINA DE INTELIGENCIA SANITARIA. REGISTRO DE CASOS REPORTADOS POR COVID 19 EN LA RED LAMBAYEQUE. CHICLAYO; 2020.
5. Son MBF. Pediatric inflammatory syndrome temporally related to covid-19. BMJ [Internet]. 3 de junio de 2020 [citado 7 de julio de 2020];369. D is pon ible e n : https://www.bmj.com/content/369/bmj.m2123

6. Health Alert Network (HAN). Provided by the Centers for Disease Control and Prevention (CDC). Multisystem Inflammatory Syndrome in Children (MIS-C) Associated with Coronavirus Disease 2019 (COVID-19) [Internet]. 2020 may [citado 7 de julio d e $\left.\begin{array}{llllll}2 & 0 & 2 & 0\end{array}\right]$. Disponible en : https://emergency.cdc.gov/han/2020/han00432 .asp

7. Sociedad La Republica. Reportan primeros casos de niños peruanos con posibles síntomas de Síndrome de Kawasaki. 1 de junio de 2020; D i s p o n i b l e e n : https://larepublica.pe/sociedad/2020/06/01/re portan-primeros-casos-de-ninos-peruanos-conposibles-sintomas-de-sindrome-de-kawasakirddr/

8. Kim Y-J, Park H, Choi YY, Kim YK, Yoon Y, Kim K-R, et al. Defining Association between COVID-19 and the Multisystem Inflammatory Syndrome in Children through the Pandemic. J Korean Med Sci [Internet]. 14 de abril de 2020 [citado 7 de julio de 2020 ]; $35\left(\begin{array}{ll}2 & 2\end{array}\right)$. Disponible en: https://doi.org/10.3346/jkms.2020.35.e204

9. Cheng MH, Zhang S, Porritt RA, Arditi M, Bahar I. An insertion unique to SARS-CoV-2 exhibits superantigenic character strengthened by recent mutations. bioRxiv. 21 de mayo de 2020;2020.05.21.109272.

10. Toubiana J, Poirault C, Corsia A, Bajolle F, Fourgeaud J, Angoulvant F, et al. Kawasaki-like multisystem inflammatory syndrome in children during the covid-19 pandemic in Paris, France: prospective observational study. BMJ [Internet]. 3 de junio de 2020 [citado 7 de julio de 2020];369. D i s p o i b l e e n : https://www.bmj.com/content/369/bmj.m2094

11. Bustos R. Síndrome inflamatorio multisistémico asociado con SARS-CoV-2 en pediatría. Rev Chil Pediatría. 2020;91(4).

12. Jones VG, Mills M, Suarez D, Hogan CA, Yeh D, Segal JB, et al. COVID-19 and Kawasaki Disease: Novel Virus and Novel Case. Hosp Pediatr. 2020;10(6):537-40.

13. Clinical Characteristics of 58 Children With a Pediatric Inflammatory Multisystem Syndrome Temporally Associated With SARS-CoV-2 I Adolescent Medicine I JAMA | JAMA Network [Internet]. [citado 24 de julio de 2020]. Disponible e $n$ : https://jamanetwork.com/journals/jama/fullar ticle/2767209

14. Plaza S, Álvarez J, Sáenz AM, Rosas M. Enfermedad de Kawasaki en paciente pediátrico en el contexto de la pandemia por COVID-19. Dermatol Venez 
[Internet]. 2020 [citado 7 de julio de 2020];58(1). D i s p o n i b l e e $n$ : http://revista.svderma.org/index.php/ojs/articl e/view/1459

15. Son MB, Fiedman K. Coronavirus disease 2019 (COVID-19): Multisystem inflammatory syndrome in children - UpToDate. Disponible en: https: / /www.uptodate.com/contents/coronavir us-disease-2019-covid-19-multisystemi n fl a m m a tory - s y n d rom e - i n children?search=sindrome $\% 20$ multisistemico $\% 20 i$ nflamatorio\%20COVID\&source=search_result\&sel
ectedTitle=1 150\&usage_type=default\&display_r ank $=1$

\section{Correspondencia}

Karen Eliana Calderón-Baldera

Correo: kareli.2092@gmail.com

\section{Revisión de pares}

Recibido: 15/06/2020

Aceptado: $30 / 06 / 2020$ 